\title{
Unicast Communication in Vehicular Ad Hoc Networks: A Reality Check
}

\author{
Mate Boban, Ozan K. Tonguz, and João Barros
}

\begin{abstract}
We characterize the unicast performance available to applications in infrastructureless vehicular ad hoc networks (VANETs) in terms of connection duration, packet delivery ratio, end-to-end delay, and jitter in both highway and urban VANET environments. The results show the existence of several stringent QoS constraints for unicast applications in infrastructureless VANETs.
\end{abstract}

Index Terms-VANET, QoS, connectivity, unicast applications.

\section{INTRODUCTION}

$\mathbf{V}$ EHICULAR Ad hoc NETworks (VANETs) have been envisioned for safety, traffic management, and commercial applications: vehicles can inform other vehicles of hazardous road conditions, traffic congestion, or sudden stops; furthermore, commercial services (e.g., data exchange, infotainment, rear-seat multiplayer games) can create an incentive for faster adoption of VANET technology.

With respect to the applications running over VANETs, Bai et al. in [1] presented a comprehensive classification of VANET applications based on their underlying network requirements. The study showed that unicast communication (both single-hop and multi-hop) will be one of the key communication paradigms required for a number of VANET applications. Apart from the unicast-based applications listed in [1], there are numerous others envisioned to be suitable for VANET environments (e.g., real time audio/video communication, instant message exchange, coordinated movement of two or more vehicles, etc.). To determine the feasibility of such applications, in this paper we determine the level of Quality of Service (QoS) that will be available in VANETs.

In order to determine achievable QoS performance in VANETs for a defined set of parameters, we implement a simulation model with the following characteristics: a) realistic implementation of PHY and MAC layers of the Dedicated Short Range Communications (DSRC) technology; b) a unicast routing scheme that exhibits optimal performance, given

Manuscript received July 18, 2009. The associate editor coordinating the review of this letter and approving it for publication was G. Karagiannidis.

This work was funded in part by the National Foundation for Science, Higher Education and Technological Development of the Republic of Croatia, under the project 03.01/34, the Fundação para a Ciência e Tecnologia (Portuguese Foundation for Science and Technology) under the Carnegie Mellon | Portugal program (Grant SFRH/BD/33771/2009), and Carnegie Mellon University.

M. Boban is with the Department of Electrical and Computer Engineering, Carnegie Mellon University, 5000 Forbes Avenue, Pittsburgh, PA, 152133890, USA, and with the Instituto de Telecomunicações, Departamento de Engenharia Electrotécnica e de Computadores Faculdade de Engenharia da Universidade do Porto, 4200-465, Porto, Portugal (e-mail: mboban@cmu.edu).

J. Barros is with the Instituto de Telecomunicações, Departamento de Engenharia Electrotécnica e de Computadores Faculdade de Engenharia da Universidade do Porto, 4200-465, Porto, Portugal.

O. K. Tonguz is with the Department of Electrical and Computer Engineering, Carnegie Mellon University, 5000 Forbes Avenue, Pittsburgh, PA, 15213-3890, USA.

Digital Object Identifier 10.1109/LCOMM.2009.091497 the underlying restrictions of the PHY and MAC layers of the DSRC standard and the dynamic nature of VANETs; and c) carefully modeled urban and highway scenarios based on real maps, vehicle densities, and speed distributions. In this letter we focus on vehicle-to-vehicle (V2V) communication, which will play a crucial role in VANETs, as infrastructure support is not envisioned to be available ubiquitously.

The main contributions of this letter are the following:

- Characterization of constraints imposed by DSRCenabled VANETs to which future unicast routing protocols and applications running over them will have to be adapted.

- Analysis of the available QoS performance (end-to-end delay, jitter, packet delivery ratio) for unicast applications in highway and urban environments; analysis of attainable connection duration for common vehicle densities in highway and urban environments.

\section{VANET ENVIRONMENT CONSIDERED}

Seeking to understand the achievable QoS in infrastructureless VANETs, we build a realistic simulation environment which assumes the optimal values of the unspecified VANET characteristics. The specified VANET characteristics include all of those that currently cannot be influenced by VANET protocol design: vehicle density, vehicle mobility, road topology, signal propagation, and DSRC as the emerging standard (we note that vehicle density and mobility could be affected by future VANET deployment). On the other hand, the routing protocols, the transport protocols, and the interference as a function of the employed routing and transport protocols, are unspecified characteristics (i.e., those that can and will be optimized by VANET research community).

To ensure optimal functioning of the routing layer, we opted for the following solution. For every message between the observed sender and receiver, all of the available paths (i.e., routes over intermediate nodes) are analyzed, and the optimal path is selected based on the minimum-delay criteria. The network layer forwards a message over each available path (if any) between the sender and receiver to determine the best path for the specific message. The process is then repeated for every message. Consequently, if the PHY and MAC layers of DSRC are able to relay the message between the sender and receiver, the network layer will deliver each message over the optimal path without additional loss or overhead. Furthermore, we employ only one sender/receiver pair per simulation run, in order to avoid the interference generated by other data sources.

Coupled together, the optimal path selection and the environment with minimal interference ensure that every message is delivered with minimum delay and without any loss 
TABLE I

SIMULATION PARAMETERS

\begin{tabular}{|c|c|}
\hline Parameter & Value \\
\hline Number of messages per sender & 20 per second \\
\hline Message size & $\begin{array}{c}\text { Normally distributed } \\
\text { mean }=100 \mathrm{~B} \text {; std. dev. }=15 \mathrm{~B}\end{array}$ \\
\hline TTL (max. \# of hops allowed) & 10 \\
\hline Vehicle densities (highway) & $10,20,40 \mathrm{veh} / \mathrm{km}$ \\
\hline Vehicle densities (urban) & $\begin{array}{l}10,50,100 \mathrm{veh} / \mathrm{km}^{2} \\
(\approx 1,5,10 \mathrm{veh} / \mathrm{km})\end{array}$ \\
\hline $\begin{array}{c}\text { Speeds of vehicles } \\
\text { Mean }\end{array}$ & $\begin{array}{l}\text { Normally distributed } \\
\text { highway }=100 \mathrm{~km} / \mathrm{h} \\
\text { urban } \approx 40 \mathrm{~km} / \mathrm{h}\end{array}$ \\
\hline Standard deviation & $\begin{array}{c}\text { highway }=25 \% \text { mean } \\
\text { urban }=35 \% \text { mean }\end{array}$ \\
\hline $\begin{array}{l}\text { Signal propagation model } \\
\text { (highway) }\end{array}$ & $\begin{array}{c}\text { Two-ray } \\
\text { (based on measurements in [5]) }\end{array}$ \\
\hline Transmission range (highway) & $550 \mathrm{~m} \mathrm{[5]}$ \\
\hline Signal propagation model (urban) & Shadowing \\
\hline Shadowing parameters & $\begin{array}{c}\quad \beta=2.6 ; \sigma_{d B}=6 \\
\text { (based on measurements in [6]) }\end{array}$ \\
\hline Noise model & Additive channel + thermal noise \\
\hline Transmission rate & $6 \mathrm{Mb} / \mathrm{s}$ \\
\hline
\end{tabular}

or overhead due to routing ${ }^{1}$. Details on the implemented routing solution can be found in [2]. The solution was implemented in the Jist/SWANS simulator with the STRAW mobility model. JiST/SWANS is a publicly available Javabased wireless network simulator. STRAW [3] is a vehicular mobility model that implements the car-following model with lane changing and intersection control. It is built on top of the JiST/SWANS platform, and constrains the node movement to real U.S. streets based on the U.S. Census Bureau's TIGER data (http://www.census.gov/geo/www/tiger).

DSRC, specified under IEEE standard $802.11 \mathrm{p}$, is becoming the de facto standard for physical (PHY) and medium access control (MAC) layers of the VANET proposed communication stack, Wireless Access in Vehicular Environment (WAVE), defined in IEEE 1609.x family of standards. Therefore, we implemented DSRC PHY and MAC layers based on the ASTM standard [4], and we used connectionless unacknowledged (Type 1) operation of the LLC as specified in the IEEE standard 802.2. We did not make use of DSRC's multi-channel functionality, since we only analyze a single sender/receiver pair sending messages to each other, and only 40 messages per second are sent in the entire system (20 messages/second in each direction between the observed pair). On top of the DSRC, we implemented the aforementioned routing solution. Simulations of highway scenarios were conducted on a $43.5 \mathrm{~km}$ long section of I-80 interstate in Elko County, $\mathrm{NV}$, whereas for the urban scenarios we used a $7.5 \mathrm{~km}^{2}$ area of downtown Chicago. We distinguish between same direction and opposite direction highway scenarios. In the same direction scenarios, the sender/receiver vehicle pair was

\footnotetext{
${ }^{1}$ Naturally, such a routing solution is not implementable in real life, since it would lack the global knowledge necessary to make the optimal path selection; nevertheless, this scenario provides us with means to analyze the optimum unicast performance achievable by DSRC-enabled VANETs.
}

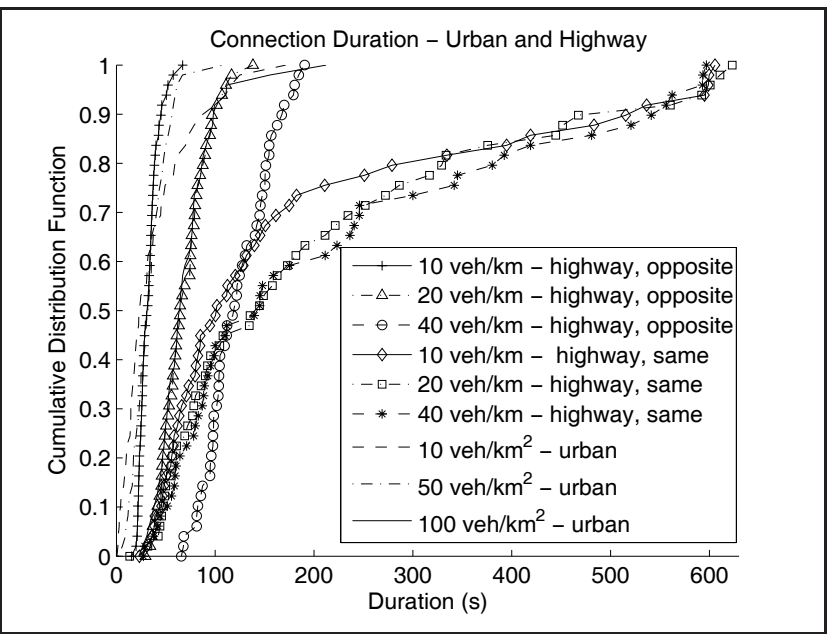

Fig. 1. The distribution of connection duration in urban and highway environments for various vehicle densities.

initially placed next to each other and proceeded to move in the same direction. In the opposite direction scenarios, the sender/receiver vehicles were placed approximately $10 \mathrm{~km}$ apart and they moved towards each other. The remaining vehicles were distributed randomly. In urban scenarios, the sender/receiver vehicles were initially placed next to each other and moved in a random direction. The remaining vehicles were distributed randomly. The effective range in urban scenarios was up to a maximum of 400 meters, with the vast majority of messages $(90+\%)$ received within the 150 meter radius. Message size and frequency were selected to model a real time unicast communication. The desired speed of vehicles for both environments was based on the normal distribution, often used in traffic engineering literature. Vehicle densities in highway scenarios were based on US DOT metrics of "low", "medium", and "high" traffic levels. Each simulation run was 900 seconds, with 200 seconds of warm-up time before any packets are sent, in order to achieve the steady state of the implemented mobility model. Simulation parameters are presented in Table I.

\section{RESULTS AND DISCUSSION}

Fig. 1 shows the connection duration as a function of vehicle density in the highway (same and opposite direction) and the urban environment. Clearly, higher vehicle densities on a highway significantly increase the connection duration for the opposite direction scenario, with the average duration increased by five times for 40 vehicles per kilometer $(\mathrm{veh} / \mathrm{km})$ compared to $10 \mathrm{veh} / \mathrm{km}$. Vehicle density does not have such a significant impact when nodes move in the same direction on highways, mostly due to the fact that the variance in the relative speed between the sender and receiver has a much greater impact than the increased number of relaying vehicles. In the urban environment, the average connection duration lies between 30 and 70 seconds. This is relatively low when compared to highway and results from the twodimensional, shadowed environment which makes continuous communication much harder to achieve. Since the observed vehicles travel in two dimensions and it cannot be assumed that they will travel in the same direction, the connection duration decreases noticeably. 


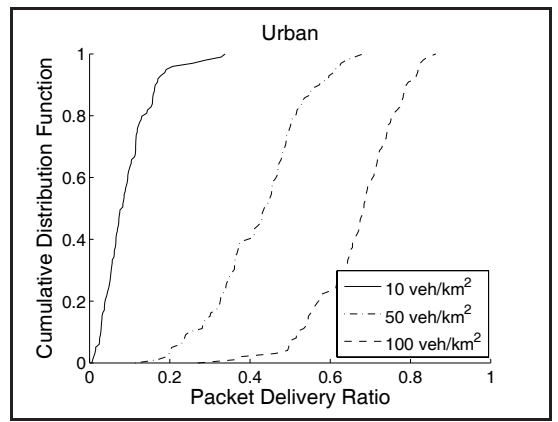

(a) Packet delivery ratio in urban environment.

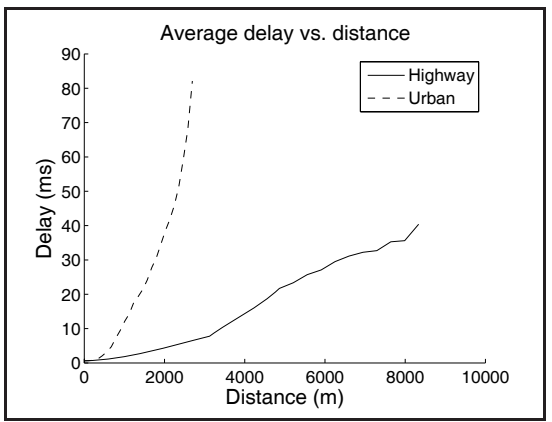

(b) End-to-end delay vs. distance.

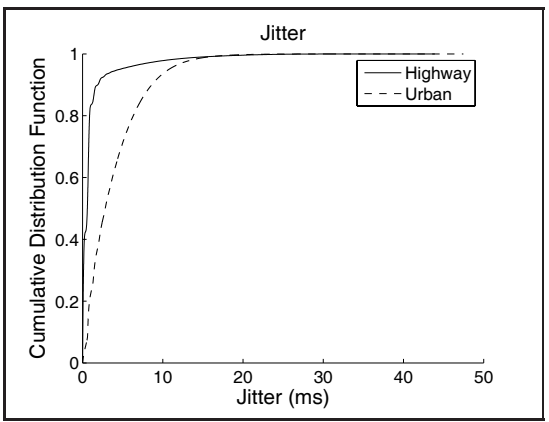

(c) The distribution of jitter.

Fig. 2. QoS (packet delivery ratio, end-to-end delay, and jitter) performance of unicast communication in an infrastructureless VANET.

Fig. 2a shows the relationship between the vehicle density and packet delivery ratio (PDR). Because the nodes in the urban environment can travel over many different roads in different directions, the existence of nodes that can relay the message over a number of hops is essential for an increase in the PDR. Mean values of PDR amount to approximately 9\%, $42 \%$, and $67 \%$ for the vehicle densities under consideration. Fig. $2 b$ shows that the end-to-end delay (which is calculated as the average end-to-end delay for the messages sent from both observed nodes) is highly dependent on the distance, and it increases fairly linearly in the highway environment, whereas the urban environment exhibits exponential increase of delay with distance, mainly due to the significantly decreased transmission range of radios, and the consequent need for larger number of hops to cover the same distance when compared to highway environment. This in turn increases the average transmission delay. Values of delay up to $40 \mathrm{~ms}$ in highway environments and up to $90 \mathrm{~ms}$ in urban environments show the capability of VANETs to support even the most delay stringent applications, but only within a certain distance. Similarly, the longer tail in the distribution of jitter in urban environment is due to the decreased transmission range, which implies a larger number of hops, thus producing larger delay variance. Fig. $2 \mathrm{c}$ shows that the jitter is largely confined to $0-10 \mathrm{~ms}$ interval in both the highway and the urban environment.

The results presented underline the capability of a DSRCenabled, infrastructureless VANET to support an end-to-end delay below $90 \mathrm{~ms}$, jitter largely below $10 \mathrm{~ms}$, and a relatively high PDR for a high enough vehicle density in an urban environment or for a small enough region of interest in a highway environment. However, applications requiring a certain connection duration (all real-time applications do) will have to be bound to a certain region of interest, since even the optimal environment implemented in this study, which is based on a single sender/receiver pair and minimum interference, clearly shows that all of the QoS metrics progressively deteriorate with distance, especially in urban environment. In a sparse network this is true because of the lack of relaying nodes: store-carry-forward routing could be used to increase the geographical range and PDR, but the delay and jitter would be exacerbated, and connection duration would be dismal. In a dense network, however, relaying the message over a large number of hops could result in flooding the network, thus significantly affecting the network performance. This is especially true for real-time applications, which create a continuous stream of messages. These results imply that over larger geographical areas infrastructure support will have to be utilized for real-time applications, in order to increase the connection duration, decrease delay, and prevent the flooding of the network.

\section{CONCLUSION}

In this letter we developed a practical methodology to study the achievable unicast performance over VANETs. To that end, we analyzed some of the most important QoS metrics for unicast communication in both highway and urban environments. The results obtained show that the proposed VANET technology has the ability to provide satisfactory delay and jitter for most applications, whereas PDR and connection duration are both highly dependent on the vehicle density and the specific environment. Moreover, the connection duration is closely related to the relative speed of vehicles. The results presented are significant in several aspects: a) they define available unicast performance over DSRC-enabled VANET in both urban and highway environments, b) they shed light on which applications could be feasible, and c) they provide insights into which applications will not be feasible in infrastructureless VANETs, given their QoS requirements.

\section{REFERENCES}

[1] F. Bai, T. Elbatt, G. Hollan, H. Krishnan, and V. Sadekar, "Towards characterizing and classifying communication-based automotive applications from a wireless networking perspective," 1st IEEE Workshop on Automotive Networking and Applications (AutoNet), 2006.

[2] M. Boban, G. Misek, and O. Tonguz, "What is the best achievable QoS for unicast routing in VANETs?" in Proc. 3rd IEEE Workshop on Automotive Networking and Applications (AutoNet), 2008, pp. 1-10.

[3] D. R. Choffnes and F. E. Bustamante, "An integrated mobility and traffic model for vehicular wireless networks," in Proc. 2nd ACM International Workshop on Vehicular Ad Hoc Networks (VANET '05), New York, pp. 69-78.

[4] "Standard Specification for Telecommunications and Information Exchange Between Roadside and Vehicle Systems - 5GHz Band Dedicated Short Range Communications (DSRC) Medium Access Control (MAC) and Physical Layer (PHY) Specifications," ASTM E2213-03, Sep. 2003.

[5] "Vehicle Safety Communications Project, Final Report," U.S. Department of Transportation, NHTSA, Crash Avoidance Metrics Partnership, Tech. Rep. DOT HS 810 591, 2006.

[6] L. Cheng, B. Henty, D. Stancil, F. Bai, and P. Mudalige, "Mobile vehicleto-vehicle narrow-band channel measurement and characterization of the $5.9 \mathrm{GHz}$ dedicated short range communication (DSRC) frequency band," IEEE J. Sel. Areas Commun., vol. 25, no. 8, pp. 1501-1516, Oct. 2007. 